\title{
Protección antiincrustante de estructuras sumergidas mediante recubrimientos formulados con 7-hidroxi-4-metilcumarina
}

\author{
Diego Ruiz ${ }^{1}$, Juan Carlos Autino ${ }^{1}$, Gustavo Romanelli ${ }^{1,2}$, Miriam Pérez ${ }^{3,4}$, \\ Mónica García ${ }^{3}$, Juan Pablo Roldán ${ }^{3}$, Guillermo Blustein ${ }^{3,5}$
}

\footnotetext{
${ }^{1}$ Centro de Investigación en Sanidad Vegetal (CISaV), Facultad de Ciencias Agrarias y Forestales, Universidad Nacional de La Plata, 60 y 119, La Plata, Buenos Aires, Argentina.

${ }^{2}$ CINDECA, UNLP-CONICET, FCE (47 No257), La Plata, Buenos Aires, Argentina.

${ }^{3}$ CIDEPINT, CICPBA-CONICET (52 e/ 121 y 122) La Plata, Buenos Aires, Argentina.

${ }^{4}$ Facultad de Ciencias Naturales y Museo, Universidad Nacional de La Plata, 122 y 60, La Plata, Buenos Aires, Argentina.

${ }^{5}$ Facultad de Ciencias Agrarias y Forestales, Universidad Nacional de La Plata, 60 y 119, La Plata, Buenos Aires, Argentina.

biofouling@ cidepint.gov.ar
}

\section{RESUMEN}

La fijación de micro y macroorganismos sobre estructuras sumergidas como embarcaciones, boyas, granjas de cultivo, plataformas offshore acarrea enormes pérdidas económicas como consecuencia del deterioro de materiales y el retiro de servicio.

Dado que las regulaciones vigentes apuntan a la utilización de productos 'amigables con el medio ambiente' para reemplazar los biocidas metálicos altamente contaminantes empleados en pinturas antiincrustantes, existe un creciente interés por hallar compuestos que resulten eficaces sin que alteren los ecosistemas.

Con esta finalidad, se sintetizó el compuesto 7-hidroxi-4-metilcumarina aplicando procedimientos de la Química Verde, esto es, en ausencia de solvente y usando ácidos sólidos recuperables, y se lo caracterizó por medio de análisis orgánicos corrientes.

En laboratorio se evaluó la actividad antiincrustante de esta cumarina mediante test de asentamiento exponiendo al mejillón Mytilus edulis platensis a distintas concentraciones. Los resultados indicaron que la cumarina fue efectiva tanto en la inhibición del asentamiento como en la producción de hebras del biso. Asimismo, se realizaron ensayos de recuperación de los organismos en agua de mar sin el agregado del compuesto comprobándose que el efecto inhibidor de la cumarina es transitorio dado que recuperaron rápidamente sus funciones vitales.

Posteriormente, se formuló una pintura de matriz soluble conteniendo este compuesto y se aplicó sobre paneles que se expusieron en el puerto de Mar del Plata por 45 y 90 días. Esta formulación demostró un marcado efecto antiincrustante sobre las especies más conspicuas de la comunidad.

El compuesto 7-hidroxi-4-metilcumarina obtenido por síntesis sustentable resulta promisorio para su utilización en pinturas antiincrustantes no solo por la eficiencia demostrada sino también por su baja toxicidad.

Palabras clave: incrustaciones biológicas, cumarinas, pinturas antiincrustantes.

\section{ABSTRACT}

Biofouling settlement on submerged structures such as ships, buoys, farms, off-shore platforms and meteorological stations induces economic losses as a consequence of material deterioration and withdraw from service.

Environmental regulations focus on the use of environmental friendly products to replace metallic biocides included in antifouling paints which are highly pollutant. A growing interest for searching and identifying efficient and benign antifoulants has being carried out.

For this reason, the compound 7-hydroxy-4-methylcoumarin was synthesized following Green Chemistry 
procedures and then, characterized by current organic analysis.

In the laboratory, antifouling activity of this coumarin was estimated by settlement test, i.e., batches of mussels (Mytilus edulis platensis) were exposed to different concentrations of the compound. Results indicated that coumarin was effective on both, settlement inhibition and byssal thread. Also, recovery tests were carried out; for this test, organisms were placed in clean seawater after coumarin exposure. It was demonstrated that coumarin effect was temporary because they could rapidly recover their vital parameters.

Then, a soluble matrix antifouling paint containing this compound was formulated. Paints were applied on panels and exposed in Mar del Plata harbour for 45 and 90 days. Painted panels with this formulation showed a marked antifouling activity on conspicuous species of the community.

Results indicated that sustainable chemistry-synthetized 7-hydroxy-4-methylcoumarin is a promising compound to be employed in antifouling paints due to its efficiency and low toxicity.

Keywords: biofouling, coumarins, antifouling coatings.

\section{INTRODUCCIÓN}

Los sustratos duros sumergidos son rápidamente colonizados por algas e invertebrados que compiten por el espacio disponible con el fin de completar sus ciclos de vida. Cuando los sustratos elegidos para el asentamiento no son sustratos naturales sino estructuras artificiales como boyas, embarcaciones, muelles, plataformas off-shore o centrales generadoras de energía, provocan graves perjuicios económicos en virtud del deterioro que ocasionan sobre los materiales, en la pérdida de eficiencia y rendimiento, en el incremento en el consumo de combustible y, a su vez, en el lucro cesante [1-2]. Esta comunidad se denomina biofouling (o incrustaciones biológicas) y está integrada por una gran diversidad de organismos como bacterias, diatomeas, mejillones, cirripedios, gusanos de tubo y ascidias representando uno de los problemas más difíciles de erradicar de todos los puertos del mundo, no solo desde el punto de vista económico sino también en lo referente a la búsqueda de métodos de control que estén acordes con el cuidado del medio ambiente [3].

La aplicación de pinturas antifouling es el método de control del biofouling más efectivo y universalmente empleado. El modo de acción de este tipo de pinturas radica en la lixiviación de compuestos biocidas que generan una interface altamente tóxica evitando la fijación de larvas y esporas algales. Sin embargo, muchos de los biocidas más efectivos como mercurio, arsénico y plomo fueron prohibidos por su alto efecto contaminante de la biota y los sedimentos. En la década del '60 se introdujeron en el mercado pinturas conteniendo organoestánnicos, como óxido de tributilestaño y trifenilestaño, con alto poder antiincrustante y larga vida útil [4]. De un modo similar, estos compuestos también fueron prohibidos debido a que se bioacumulan, no son biodegradadables y son tóxicos tanto para organismos del fouling como para especies non-target [5]. Este panorama llevó a la búsqueda de nuevos productos igualmente efectivos pero "amigables con el medio ambiente". En este sentido, muchos organismos marinos como esponjas, corales y tunicados surgieron como fuente de compuestos antifouling debido a que naturalmente desarrollan defensas químicas para evitar ser colonizados por otras especies. Básicamente se aislaron e identificaron terpenoides, esteroides, carotenoides, compuesto fenólicos, furanonas, alcaloides, péptidos y lactonas [6].

Por otra parte, ciertos metabolitos secundarios de plantas terrestres demostraron actividad antiincrustante [7]. Entre los metabolitos más comunes obtenidos de plantas se encuentran las cumarinas, benzo $\alpha$ pironas derivadas del metabolismo de la fenilalanina. Las cumarinas se encuentran en gran cantidad en aceites esenciales, principalmente en la corteza de canela, hojas de casia y de lavanda, en frutos (moras, arándanos), té sustentable y otros alimentos como la achicoria. La presencia del anillo cumarínico en diversos productos es responsable de muchas propiedades biológicas como por ejemplo, actividad anticoagulante, estrogénica, antimicrobiana, antifúngica, vasodilatora, moluscicida y antihelmíntica. [8-9]. Si bien algunas cumarinas son compuestos tóxicos, la 7-hidroxi-4-metilcoumarina es un compuesto seguro que se emplea como ingrediente activo en muchas drogas que ya han sido aprobadas [10]. Por otra parte, son compuestos degradables por bacterias y hongos [11]. A pesar de que muchas cumarinas naturales pueden ser aisladas de plantas superiores y microorganismos, la síntesis en laboratorio por medio de la aplicación de técnicas de "síntesis sustentable" es posible incluyendo procesos, reactivos y técnicas seguras [12-14]. Por esta razón, las cumarinas obtenidas por síntesis sustentable resultan candidatas promisorias para estudiar como compuestos antifouling. El objetivo de este trabajo es evaluar las propiedades antiincrustantes de la 7-hidroxi-4-metilcumarina (Figura 1) sobre el mejillón Mytilus edulis platensis en el laboratorio e incluida en una pintura antifouling en ensayos en el mar. 


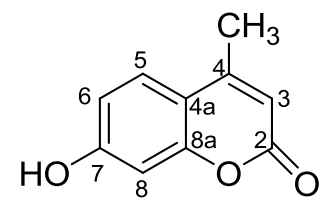

Figura 1: Estructura de 7-hidroxi-4-metilcumarina

El método más corriente para preparar cumarinas sustituidas en la posición 4 es la reacción de Pechmann, que toma como punto de partida materiales simples como fenoles y $\beta$ cetoésteres o ácidos $\beta$ carboxílicos insaturados. La reacción incluye catálisis ácida y pueden obtenerse buenos rendimientos de cumarinas. Las cantidades de mineral ácido en bruto que usualmente se requieren en las preparaciones clásicas llevan a incrementar la polución ambiental y se enfatiza esta cuestión para disminuir el impacto, por ejemplo, en química orgánica clásica se utiliza $1,1 \mathrm{~L}$ de $\mathrm{H}_{2} \mathrm{SO}_{4}$ para preparar 1 mol de 7-hidroxi-4-metilcoumarina por la reacción de Pechmann [13]. Recientemente, se ha puesto énfasis en disminuir el impacto ambiental de los procesos químicos. La amplia utilidad de los heteropoliácidos (HPAs) como ácidos y catalizadores oxidativos en solución así como en el estado sólido para varios procesos industriales fue demostrada para una amplia variedad de transformaciones sintéticas útiles de sustratos.

\section{MATERIALES Y MÉTODOS}

\subsection{Síntesis sustentable de 7-hidroxi-4-metilcumarina}

Se emplearon drogas provistas por Aldrich y Fluka y se utilizaron inmediatamente después de su purificación por procedimientos estándar (destilación y recristalización). Las reacciones fueron monitoreadas por TLC sobre placas de sílica gel prerecubiertas $(254 \mathrm{~mm})$ y todos los rendimientos se calcularon a partir de productos cristalizados. El producto fue identificado por comparación con datos físicos publicados (pf, TLC y RMN) o con muestras auténticas preparadas con los respectivos métodos convencionales usando ácido sulfúrico como catalizador. El punto de fusión del compuesto se determinó en un tubo capilar sellado.

El espectro de RMN fue obtenido en un espectrofotómetro NMR Bruker Advance DPX 400 como soluciones d6-DMSO y los corrimientos químicos se expresaron en unidades $\delta$ con $\mathrm{Me}_{4} \mathrm{Si}$ (TMS) como patrón interno. El catalizador $\mathrm{H}_{14} \mathrm{NaP}_{5} \mathrm{~W}_{30} \mathrm{MoO}_{110}$ fue sintetizado de acuerdo con procedimientos reportados previamente [15]. La 7-hidroxi-4-metilcoumarina fue sintetizada según la metodología de la Química Verde [16] por medio de la reacción de Pechmann a partir de una mezcla de resorcinol $(10 \mathrm{mmol})$ y acetoacetato de etilo $(10 \mathrm{mmol})$ en ausencia de solventes. La mezcla fue agitada a $130^{\circ} \mathrm{C}$ en presencia de un heteropoliácido de Preyssler $\mathrm{H}_{14} \mathrm{NaP}_{5} \mathrm{~W}_{30} \mathrm{O}_{110}(1 \% \mathrm{mmol})$ durante $1 \mathrm{~h}$ en un balón que fue equipado con un refrigerante e inmerso en un baño de aceite. La mezcla reactiva fue extraída con tolueno a $110^{\circ} \mathrm{C}(3 \mathrm{x} 5 \mathrm{~mL})$, la solución fue concentrada y el producto crudo recristalizado a partir de metanol. El producto puro fue caracterizado por su punto de fusión y espectros ${ }^{13} \mathrm{C}$ RMN, ${ }^{1} \mathrm{H}$ RMN, IR y MS.

\subsection{Colecta de organismos}

Para la realización de los ensayos de laboratorio se colectaron mejillones de la especie Mytilus edulis platensis en el sustrato rocoso en la zona de Playa Chica (38 $\left.08^{\prime} 17^{\prime \prime} \mathrm{S}, 57^{\circ} 31^{\prime} 18^{\prime \prime}\right)$. Estos bivalvos fueron elegidos dado que son integrantes relevantes del fouling del puerto de Mar del Plata. En el laboratorio los mejillones fueron separados unos de otros y acondicionados en agua de mar artificial (ASTM D1141/75), pH 8,2-8,3, salinidad 33-35\%, con una temperatura de $22 \pm 2^{\circ} \mathrm{C}$, suficiente aireación y luz natural.

\subsection{Ensayos con mejillones}

Una vez obtenida la 7-hidroxi-4-metilcoumarina se preparó una solución madre disolviendo $300 \mathrm{mg}$ del compuesto en $10 \mathrm{~mL}$ de metanol. A partir de esta solución madre $(30 \mathrm{mg} / \mathrm{mL})$ se obtuvieron ocho diluciones: 3,$0 ; 2,1 ; 1,4 ; 0.9 ; 3,6 ; 0,3 ; 0,03$ y $0,003 \mathrm{mg} / \mathrm{mL}$. Luego se tomó $1 \mathrm{~mL}$ de cada solución, se lo colocó en una cápsula de Petri de $9 \mathrm{~mm}$ de diámetro y se dejó evaporar el solvente a temperatura ambiente. Se colocaron los mejillones y se evaluó la producción de hilos del biso para cada concentración de cumarina siguiendo la metodología propuesta por Ina et al. [17]. A fin de estimar la actividad antifouling se realizaron ensayos utilizando grupos de seis mejillones (entre 1,0 y 1,5 cm de longitud) que fueron fijados al fondo de la cápsula de Petri con adhesivo de dos componentes (Poxipol@); el agua de mar no fue cambiada ni los mejillones alimentados durante los experimentos. La cumarina fue ensayada en las siguientes concentraciones: 0,047; 0,47; 
4,$7 ; 9,4 ; 14,0 ; 22,0 ; 3,0$ y 47,0 mg/ $\mathrm{cm}^{2}$. Luego, los recipientes se completaron con agua de mar artificial. Para el control se adicionó $1 \mathrm{~mL}$ de solvente y se dejó evaporar. Todos los ensayos se realizaron por triplicado en condiciones de oscuridad y a $14-18^{\circ} \mathrm{C}$.

\subsubsection{Inhibición de la producción de hilos del biso}

Este experimento se llevó a cabo para determinar la concentración necesaria de cumarina para inhibir el 50\% del número de hilos del biso luego de 24 horas. El conteo de los bisos se realizó bajo estereomicroscopio, previa tinción con fucsina.

\subsubsection{Ensayos de asentamiento}

Se registran número de individuos asentados y no asentados en diferentes concentraciones de cumarina luego de 24 horas. Se determina la concentración inhibitoria del asentamiento para la mitad de los individuos en ensayo $\left(\mathrm{EC}_{50}\right)$.

\subsubsection{Test de recuperación}

Para este test todos los individuos expuestos a las distintas concentraciones de cumarina y sus réplicas (adheridos o no) se transfirieron a agua de mar artificial limpia durante 24 horas. Luego, se registró el número de hilos producidos y la cantidad de individuos adheridos a fin de estimar el porcentaje de recuperación y determinar el grado de toxicidad del compuesto.

\subsection{Ensayos de campo}

Se prepararon pinturas antifouling utilizando resina colofonia como ligante y ácido oleico como plastificante. La pintura fue preparada a escala de laboratorio en un molino de bolas en jarras de $1 \mathrm{~L}$ en condiciones operativas adecuadas para asegurar una buena dispersión. Se disolvieron conjuntamente la resina colofonia y el ácido oleico en una mezcla solvente de xileno/metil isobutil cetona (1:1) usando una dispersora de alta velocidad. Luego el molino fue cargado con esta mezcla ("vehículo"), los pigmentos (óxido de cinc y carbonato de calcio) y dispersado durante 24 horas. Luego la pintura fue filtrada y fraccionada en dos porciones, una de las cuales fue utilizada como control negativo y la restante como tratamiento. En este último caso, la 7hidroxi-4metilcumarina fue previamente disuelta en $1 \mathrm{~mL}$ de metanol e incorporada a la matriz de pintura al $2 \%$ en peso y dispersada durante una hora (Tabla 1). Las pinturas antifouling fueron aplicadas sobre paneles de acrílico $(4 \times 12 \mathrm{~cm})$, previamente arenados y desengrasados con tolueno. Se aplicaron cuatro capas de pintura con tiempos de secado de 24 horas entre manos hasta obtener un espesor final de película seca de $75 \pm$ $5 \mu \mathrm{m}$. Los paneles se colocaron en bastidores de aluminio y se sumergieron en el puerto de Mar del Plata. Como control se utilizaron paneles limpios y paneles pintados con pintura base (sin cumarina). Las inspecciones de campo se realizaron a los 45 y 90 días de exposición durante el verano (diciembre-marzo) cuando el asentamiento del fouling es más abundante. Este asentamiento fue estimado empleando una grilla de puntos al azar.

En todos los casos se aplicaron test estadísticos y se realizaron los experimentos por triplicado. Las diferencias entre controles y tratamientos fueron determinadas por ANOVA seguido del test LSD. Las diferencias se consideraron significativas para $\mathrm{p}<0.05$; las estimaciones de $\mathrm{EC}_{50} \mathrm{y}$ reducción del $50 \%$ de producción de hilos del biso fueron calculadas con análisis Probit.

Tabla 1: Composición de las pinturas expresadas como porcentaje en peso

\begin{tabular}{l|c|c}
\hline COMPONENTES & CONTROL & TRATAMIENTO \\
\hline óxido de cinc & 43.9 & 41.9 \\
\hline carbonato de calcio & 14.1 & 14.1 \\
\hline colofonia & 17.1 & 17.1 \\
\hline ácido oleico & 2.9 & 2.9 \\
\hline 7-hidroxi-4-metilcumarina & ----- & 2.0 \\
\hline xileno/metil isobutil cetona (1:1) & 22.0 & 22.0 \\
\hline
\end{tabular}




\section{RESULTADOS}

\subsection{Caracterización de la 7-hidroxi-4metilcumarina}

El procedimiento descripto previamente provee una alternativa útil, limpia y rápida para la preparación de la 7-hidroxi-4-metilcumarina obteniéndose $92 \%$ de rendimiento del producto puro. El tiempo de reacción puede ser reducido entre 7 y 8 veces en comparación con los métodos clásicos [18].

Punto de fusión: $186-187^{\circ} \mathrm{C}$ (literatura. p.f.: $185^{\circ} \mathrm{C}$ [18])

${ }^{13} \mathrm{C}$ RMN (DMSO-d6, $\left.100 \mathrm{MHz}\right) \delta$ (ppm): 162.5 (C-2 ó C-7), 161.8 (C-2 ó C-7), 155.3 (C-8a), 155.0 (C-4), 127.6 (C-5), 114.3 (C-4a), 113.1 (C-6), 110.9 (C-3), $103.2(\mathrm{C}-8), 19.0\left(\mathrm{CH}_{3}\right)$.

${ }^{1} \mathrm{H}$ RMN (DMSO d6, $\left.400 \mathrm{MHz}\right) \delta$ (ppm): 10.53 (1H, s, OH), 7.45 (1H, d, J: 8.8, H-5), 6.70 (1H, dd, J: 8.8, J: 2.3, H-6), 6.58 (1H, d, J: 2.3, H-8), 5.99 (1H, d, J: 1.1, H-3), 2.24 (3H, d, J: 1.0, $\left.\mathrm{CH}_{3}\right)$.

Espectro de masas: m/z: $176\left(\mathrm{M}^{+}, 70 \%\right), 148$ (100\%), 147 (49\%), $92(15 \%)$.

Espectro IR (KBr, $\left.\mathrm{cm}^{-1}\right): 3080(\mathrm{O}-\mathrm{H}), 1690(\mathrm{C}=\mathrm{O}), 1610(\mathrm{O}-\mathrm{H})$.

\subsection{Ensayos con mejillones}

\subsubsection{Inhibición de la producción de hilos del biso}

Los mejillones se encuentran entre los organismos más conspicuos del fouling y pueden ser utilizados como bioindicadores para estudios antifouling [19]. Las cumarinas inhibieron significativamente la adhesión de los hilos del biso respecto del control demostrando una fuerte actividad antifouling. Los bioensayos indicaron que la reducción del $50 \%$ en la producción del número de bisos fue obtenida a una concentración de 0,157 $\mu \mathrm{g} / \mathrm{cm}^{2}(\mathrm{p}<0,05)$. Sin embargo, la bisogénesis fue afectada desde valores tan bajos como $0,047 \mu \mathrm{g} / \mathrm{cm}^{2}$. No obstante, la producción de hilos fue marcadamente afectada en concentraciones superiores a $22 \mu \mathrm{g} / \mathrm{cm}^{2}$.

\subsubsection{Ensayos de asentamiento}

La concentración efectiva de cumarina para la inhibición del asentamiento de la mitad de la población de mejillones $\left(\mathrm{EC}_{50}\right)$ fue de $11 \mu \mathrm{g} / \mathrm{cm}^{2}$. Existen muy pocos reportes [20-21] acerca de la actividad antifouling de las cumarinas y, en todos los casos, los tests fueron llevados a cabo solamente a escala de laboratorio. Se ha reportado que los extractos metanólicos conteniendo cumarinas obtenidas de macroalgas fueron capaces de inhibir completamente la producción de hilos del biso en el mejillón Perna indica [19].

\subsubsection{Test de recuperación}

La cantidad producida de hilos del biso no fue afectada por la exposición a la cumarina en la mayoría de las concentraciones, es decir, no existen diferencias en la producción entre organismos tratados y no tratados indicando que la exposición al compuesto no causó ningún daño irreversible.

\subsection{Ensayos de campo}

Las principales especies del micro y macro fouling del puerto de Mar del Plata fueron inhibidas por las pinturas conteniendo cumarinas. Particularmente, luego de 45 días de inmersión se detectaron diferencias significativas en el asentamiento de las algas Enteromorpha intestinalis y Ectocarpus sp., en colonias de briozoos (Bugula stolonifera), gusanos tubícolas (Polydora sp. y Hydroides elegans) y ascidias (Ciona intestinalis), manteniéndose el poder antiincrustante durante los 90 días de experimentación (p < 0.05) (Figuras 2 y 3). 


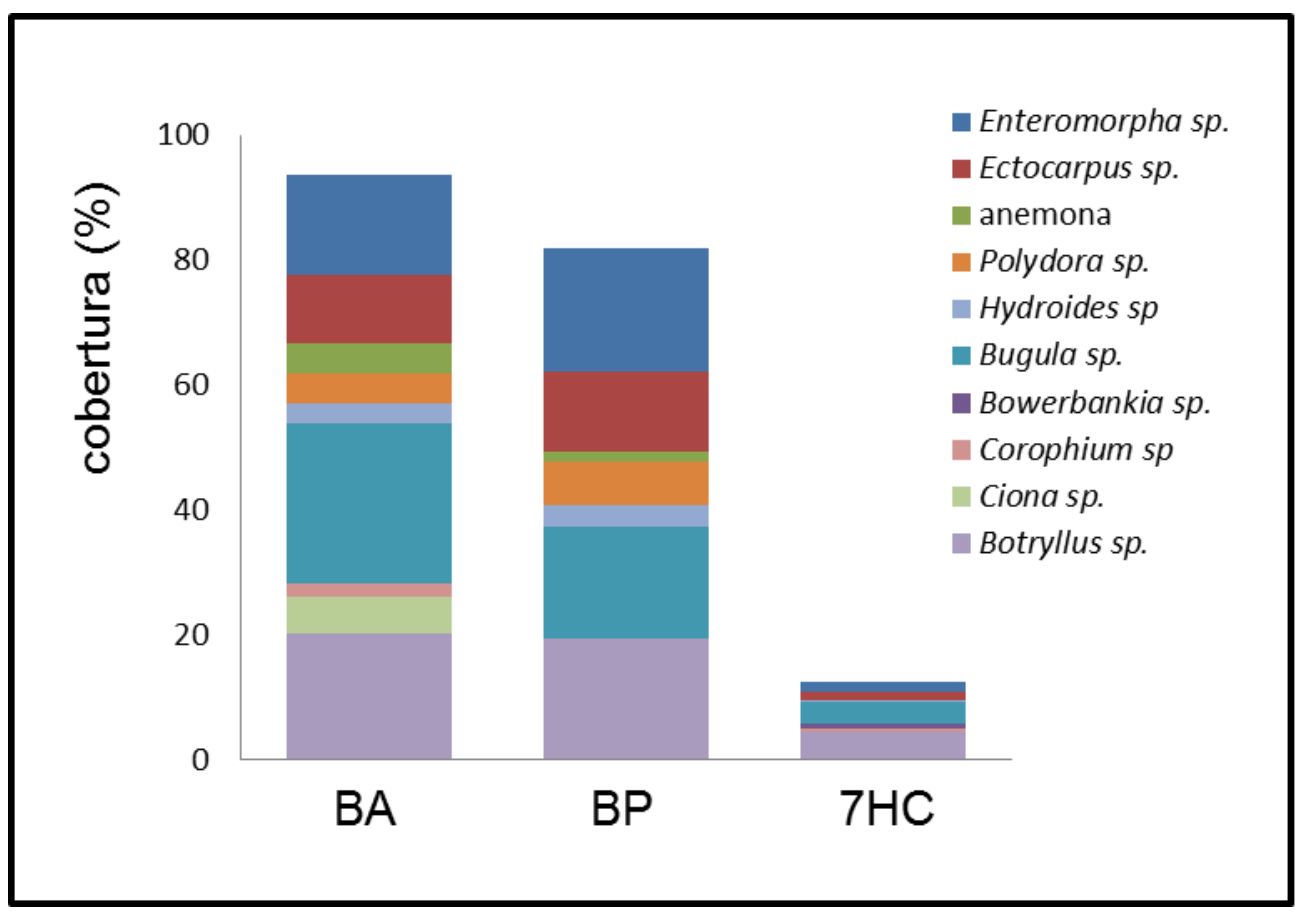

Figura 2: Porcentajes de cobertura de macrofouling asentado por especies sobre paneles pintados vs. control, 45 días de exposición BA: panel de acrílico, BP: pintura base, 7HC: pintura antifouling con 7-hidroxi-4-methilcoumarina. Barras= media $\pm \mathrm{SE},(*)$ diferencias significativas, $\mathrm{p}<0.05$.

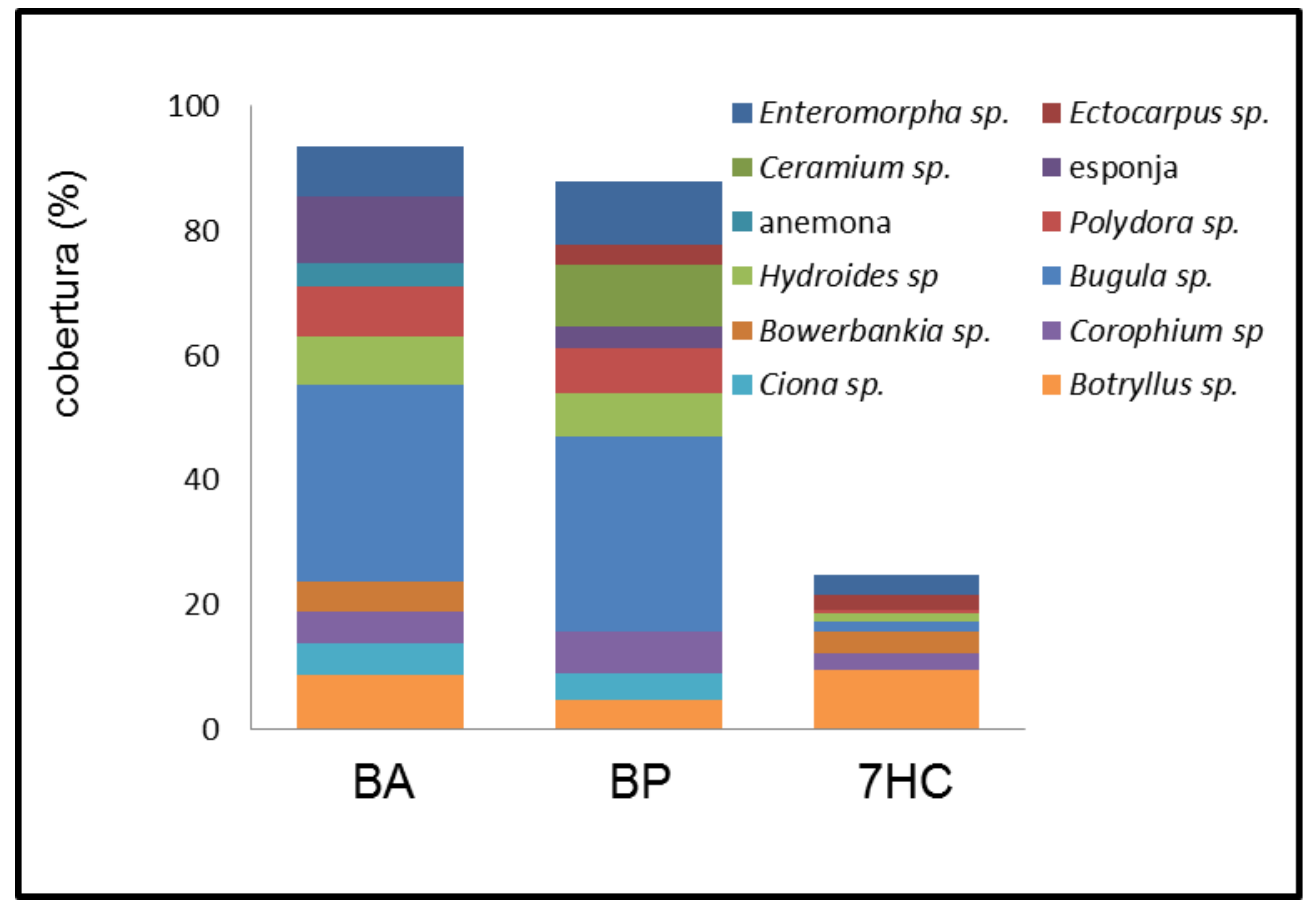

Figura 3: Porcentajes de cobertura de macrofouling asentado por especies sobre paneles pintados vs. control, 90 días de exposición. BA: panel de acrílico, BP: pintura base, 7HC: pintura antifouling con 7-hidroxi-4-methilcoumarina. Barras= media $\pm \mathrm{SE},(*)$ diferencias significativas, $\mathrm{p}<0.05$. 
Los resultados de estos experimentos concuerdan con los hallados para cumarinas aisladas del árbol de canela que evitaron el asentamiento de algas [20] y de cumarinas de hierbas que mostraron efecto antiincrustante sobre briozoos y cirripedios [21]. Las propiedades de las cumarinas fueron intensamente estudiadas en relación con su estructura molecular. En particular, las sustituciones con oxígeno son esenciales para la actividad antifúngica [22] y la posición de la función polar $(\mathrm{OH})$ o menos polar $(\mathrm{OMe}, \mathrm{Me})$ están asociadas con la actividad antibacteriana [23]. Una propiedad típica de las cumarinas es su carácter lipofílico, que sumado a su estructura molecular plana, influye en ciertas actividades biológicas como por ejemplo la posibilidad de ingresar a la célula por difusión pasiva y actuar a menor concentración [24]. Las cumarinas afectarían la formación del biofilm y, en consecuencia, la secuencia de formación del biofouling. A su vez, la elevada lipofilia reduciría su solubilidad favoreciendo la posibilidad de incorporación a una pintura antifouling y regulando su lixiviación en el agua.

El uso del heteropoliácido de Preyssler $\mathrm{H}_{14} \mathrm{NaP}_{5} \mathrm{~W}_{30} \mathrm{O}_{110}$ como catalizador, combinado con condiciones libres de solvente es una alternativa prometedora para la síntesis de derivados de la cumarina. Las características sobresalientes del anión de Preyssler son su disponibilidad, reutilización e inocuidad. Entre las "ventajas sustentables" del procedimiento descripto se encuentran la baja producción de desechos, la reducción del tiempo de reacción, la no utilización de solventes y adsorbentes y, principalmente, el reemplazo de ácidos minerales corrosivos. La síntesis orgánica permite obtener un gran número de moléculas bioactivas o mejorar la actividad de compuestos conocidos sobre la base de "grupos clave" presentes en el producto natural que podrían estar involucrados en la actividad antifouling. El desarrollo de una síntesis adecuada de estas moléculas en el laboratorio es importante porque es a menudo prácticamente imposible extraer suficiente cantidad de compuesto de los organismos vivos para cubrir las necesidades de la industria antifouling.

La síntesis total en el laboratorio de la familia de las cumarinas por medio de técnicas de Química Verde toma un rol importante en el cuidado del ambiente para la producción de nuevos antifoulants. La alternativa de la síntesis sustentable es urgente para la industria de las pinturas marinas y es de gran interés en el desarrollo de recubrimientos libres de biocidas.

\section{CONCLUSIONES}

Los experimentos de laboratorio y de campo demuestran que la cumarina empleada, obtenida mediante "síntesis sustentable" tiene actividad antifouling sobre un gran espectro de organismos de la comunidad incrustante cuyo asentamiento ocasiona graves perjuicios a las estructuras sumergidas. Otra ventaja que presenta es que la actividad antifouling se observa desde concentraciones superficiales muy bajas $\left(50 \mathrm{ng} / \mathrm{cm}^{2}\right)$ y tiene un efecto reversible, es decir, los organismos recuperan su actividad normal una vez trasladados a agua de mar sin el producto.

En conclusión, 7-hidroxi-4-metilcoumarina resulta un excelente candidato como aditivo de baja toxicidad para pinturas marinas.

\section{AGRADECIMIENTOS}

Los autores agradecen al CONICET, CICPBA y UNIVERSIDAD NACIONAL de LA PLATA por el apoyo brindado para realizar este trabajo. Asimismo se agradece al Club Motonáutico de Mar del Plata por ceder sus instalaciones para realizar los ensayos de campo.

\section{BIBLIOGRAFÍA}

[1] HELLIO, C., TSOUKATOU, M., MARECHAL, J., et al., "Inhibitory effects of mediterranean sponge extracts and metabolites on larval settlement of the barnacle Balanus amphitrite", Marine Biotechnology, v.7, pp. 297-305, 2005.

[2] DOBRETSOV, S., ABED, R., TEPLITSKI, M., "Mini-review: inhibition of biofouling by marine microorganisms", Biofouling, v. 29, pp. 423-441, 2013.

[3] DOBRETSOV, S., DAHMS H., P.Y. QIAN, "Inhibition of biofouling by marine microorganisms and their metabolites", Biofouling, v. 22 pp. 43-54, 2006.

[4] IWAO, O. "Organotin antifouling paints and their alternatives", Applied Organometallic Chemistry, v. 17 pp. 81-105, 2003.

[5] YEBRA, D., KIIL S., DAM-JOHANSEN, K. “Antifouling technology: past, present and future steps towards efficient and environmentally friendly antifouling coatings", Progress in Organic Coatings, v. 50, pp. 75-104, 2004. 
[6] RAVEENDRAN T., LIMNA MOL, V. "Natural product antifoulants", Current Science, v. 97, pp. 508520, 2009.

[7] PÉREZ, M., GARCÍA, M., SÁNCHEZ, M., et al., "Effect of secochiliolide acid isolated from the Patagonian shrub Nardophyllum bryoides as active component in antifouling paints", International Biodeterioration \& Biodegradation, v. 89, pp. 37-44, 2014.

[8] ASMA'A, A., MIKDAD, A., ASHOK, S., et al., "Synthesis, characterization, and antimicrobial activity of some new coumarin derivatives", Medicinal Chemical Research, v. 21, pp. 468-476, 2012.

[9] SOINE, T., "Naturally occurring coumarins and related physiological activities", Journal of Pharmaceutical Sciences, v. 53, pp. 231-264, 1964.

[10] NAGY, N., KUIPERS, H., FRYMOYER, A., et al., "4-Methylumbelliferone treatment and hyaluronan inhibition as a therapeutic strategy in inflammation, autoimmunity, and cancer", Frontiers in Immunololgy, v. 6, pp. 1-11, 2015.

[11] NIGAM, S. "Microbial biotransformation- A novel approach for modification on coumarin substrates", Indian Journal of Biotechnology, v. 12, pp. 379-385, 2013.

[12] SHELDON, R., ARENDS, I., HANEFELD, U. Green chemistry and catalysis, Wiley-VCH, 2007.

[13] TROST, B. "The atom economy: a serch for synthetic efficiency", Science, v. 254, pp. 1471-1477,1991.

[14] ANASTAS, P., WARNER, J. Green Chemistry: Theory and Practice, Oxford University Press, 1998.

[15] PASQUALE, G., RUIZ, D., JIOS, J., et al., "Preyssler catalystpromoted rapid, clean, and efficient condensation reactions for 3H-1,5- benzodiazepine synthesis in solvent-free conditions", Tetrahedron Letters, v. 54, pp. 6574-6579, 2013.

[16] ROMANELLI, G., BENNARDI, D., RUIZ, D., et al., "A solvent-free synthesis of coumarins using a Wells-Dawson heteropolyacid as catalyst", Tetrahedron Letters, v. 45, pp. 8935-8939, 2004.

[17] INA, K., TAKASAWA, R., YAGI, A., et al., "An improved assay method for antifouling substances using the blue mussel, Mytilus edulis", Agricultural and Biological Chemistry, v. 53, pp. 3319-3321, 1989.

[18] FURNISS, B., HANNAFORD, A., ROGERS, V., et al., "4-Methylcoumarin", Vogel's Textbook of Practical Organic Chemistry, Longman, Londres, pp. 924-925, 1978.

[19] IYAPPARAJ, P., RAMASUBBURAYAN, R., RAMAN, T., et al., "Evidence for the antifouling potentials of marine macroalgae Sargassum wightii". Advances in Natural and Applied Sciences, v. 6, pp. 153-162, 2012.

[20] KIM, Y., SHIN, H., CHO, J. "Antifouling activity of coumarin and its derivatives isolated from the cinnamon tree Cinnamomum loureiroi". Korean Journal of Fisheries and Aquatic Sciences, v. 46, pp. 53-58, 2013.

[21] WANG, Z., FENG, D., KE, C. "Coumarins from the herb Cnidium monnieri and chemically modified derivatives as antifoulants against Balanus albicostatus and Bugula neritina larvae". International Journal of Molecular Sciences, v. 14, pp. 1197-1206, 2013.

[22] ARAÚJO, R., GUERRA, F., LIMA, O., et al., "Synthesis, structure-activity relationships (SAR) and in silico studies of coumarin derivatives with antifungal activity". International Journal of Molecular Sciences, v. 14, pp. 1293-1309, 2013.

[23] SOUZA, S., DELLE MONACHE, F., SMANIA JR., A. "Antibacterial activity of coumarins". Zeitschrift für Naturforschung, v. 60, pp. 693-700, 2005.

[24] XU, Y. "Antifouling compounds from deep-sea bacteria and their potential mode of action", Ph.D. Thesis, Hong Kong University of Science and Technology, 2009. 